\title{
IVUS Findings of Drug Eluting Balloon Failure in the Treatment of In-Stent Restenosis
}

\section{Sung Yun Lee ${ }^{1 *}$, Sang-Wook Kim ${ }^{2}$, Jin Bae Lee ${ }^{3}$, Eun-Seok Shin ${ }^{4}$, Joon Hyung Doh', Young Joon Hong ${ }^{5}$, Jae-Jin Kwak ${ }^{1}$ and Hyung Yoon Kim ${ }^{5}$}

${ }^{1}$ Division of Cardiology, Department Internal Medicine, Cardiovascular Center, Inje University Ilsan-Paik Hospital, South Korea

${ }^{2}$ Chung-Ang University Hospital, Seoul, South Korea

${ }^{3}$ Daegu Catholic University Hospital, South Korea

${ }^{4}$ Ulsan University Hospital, South Korea

${ }^{5}$ Chonnam National University Hospital, South Korea

*Corresponding author: Sung Yun Lee, Division of Cardiology, Department Internal Medicine, Cardiovascular Center, College of Medicine, Inje University Ilsan-Paik Hospital, 170 Joohwa-ro, Ilsanseo-gu, Goyang-si, Gyeonggi-do10380, South Korea, Tel: +82-31-910-7830; +82-31-910-7927, Fax: +82-31-910-7829

\begin{abstract}
Background and objective: The objective of this study is to investigate pre-intervention IVUS characteristics of instent restenosis (ISR) lesion correlated with PEB failure.

Materials and methods: We performed pre-intervention IVUS for 58 patients with ISR and treated with PEB angioplasty. The PEB failure was defined as death, myocardial infarction and symptom driven revascularization. IVUS images were analyzed at 6 locations: Proximal and distal vessel segment within $3 \mathrm{~mm}$ from stent edge, proximal and distal stent edge, lesion site at minimal lumen area and minimal stent area.
\end{abstract}

Results: Among 58 patients treated with 58 PEB (3.0 \pm $0.4 \mathrm{~mm}$ by $21.9 \pm 4.8 \mathrm{~mm}$ ), PEB failure were developed at 8 patients $(13.8 \%)$ during 0.81 years of follow-up. ISR of drug eluting stent comprised large proportion (86.2\%) among index procedure. There was no significant difference in clinical presentation and used stents at index procedure between PEB failure and non-failure group. In PEB failure group, neointimal area $\left(4.0 \pm 0.7\right.$ vs. $2.9 \pm 0.8 \mathrm{~mm}^{2}, \mathrm{p}=$ $0.05)$ and neointimal hyperplasia (\%) (59.4 \pm 12.0 vs. 46.0 $\pm 24.9 \%, p=0.05$ ) were significant greater than non-failure group. Stent under-expansion was found in 24 ISR lesions $(48.0 \%)$ of non-failure group, whereas none was detected in PEB failure group.

Conclusion: PEB failure for ISR lesion could be associated with prominent neointimal hyperplasia on normally extended stent. Future studies are warranted to investigate another treatment modality that might substitute PEB in the treatment of ISR lesions with those characteristics.

\section{Keywords}

Intravascular ultrasound (IVUS), Coronary restenosis, Neointimal hyperplasia, Drug-eluting balloon (DEB), Paclitaxel

\section{Introduction}

In-stent restenosis (ISR) remains unsolved issue in the field of interventional cardiology, though drug-eluting stents (DES) has markedly reduced the occurrence. There is no gold standard in the treatment of ISR, drug-eluting balloons (DEB) has emerged as alternative interventional device to DES.

Paclitaxel-eluting balloons (PEB) angioplasty has been reported to be superior to plain old balloon angioplasty (POBA) and non-inferior to paclitaxeleluting stents (PES) implantation for treatment of bare metal stent (BMS) restenosis [1-6]. PEB angioplasty has been shown to be superior to POBA for treatment of DES restenosis $[7,8]$. However, an incidence of target lesion revascularization rate has been reported as $10 \%$ at 12 or 24 months after PEB angioplasty (range 4 to 20\%) [9-11]. Although PEB has shown notable clinical outcomes in recent trials, no sufficient information

Citation: Lee SY, Sang-Wook K, Lee JB, Eun-Seok S, Doh JH, et al. (2018) IVUS Findings of Drug Eluting Balloon Failure in the Treatment of In-Stent Restenosis. Int J Clin Cardiol 5:133. doi.org/10.23937/23782951/1410133

Accepted: December 22, 2018; Published: December 24, 2018

Copyright: (C) 2018 Lee SY, et al. This is an open-access article distributed under the terms of the Creative Commons Attribution License, which permits unrestricted use, distribution, and reproduction in any medium, provided the original author and source are credited. 
exists on the underlying mechanisms of treatment with PEB. Intravascular ultrasound (IVUS) can be used to identify mechanisms of ISR, for example, intimal hyperplasia, under-expansion of stent and geographic miss such as edge problem [12].

To our knowledge, there is no systemized report for IVUS characteristics of PEB failure in the treatment of ISR. The objectives of this study were to investigate preintervention IVUS characteristics of ISR lesion correlated with PEB failure and further to suggest as predictors of clinical outcomes.

\section{Materials and Methods}

\section{Study design and patients}

In this multicenter prospective observational study, we evaluated patients with newly detected ISR on follow-up coronary angiography among patients who previously have received coronary stent. The diagnosis of ISR was determined by $>50 \%$ of percent diameter stenosis at coronary angiogram. We excluded patients with left main disease and graft vessel disease and lesions that were pre-dilated before IVUS, lesions treated with stent implantation by operator's decision and follow up duration less than 6 months.

Patients were categorized into 2 groups according to the presence or absence of event after PEB angioplasty treatment: Non-PEB failure group vs. PEB failure group.

This study was notified and approved by the institutional ethics committee, and all patients provided written informed consent.

\section{Data collection}

Baseline clinical data were obtained by an experienced research nurse at the time of the procedure. Demographic and clinical information included gender, age, presence of hypercholesterolemia (under treatment or < $240 \mathrm{mg} / \mathrm{dl}$ ), diabetes mellitus (dietary glycemic control or oral hypoglycemic agent or insulin-treated), hypertension. Data on previous coronary intervention included clinical presentation (angina or myocardial infarction), stent type, stent diameter and length. All patients were interviewed at clinics and contacted by phone on clinical status minimum 6 months after the procedure.

\section{Interventional procedure}

Patients who had ISR lesion on conventional coronary angiography underwent PEB (SeQuent Please ${ }^{\circledR}$, B Braun, Melsungen, Germany) angioplasty. The technique for the ISR lesion treatment was a stepwise strategy according to the "stenting" approach. Before PEB angioplasty, we performed pre-PCI IVUS and subsequent plaque or stent modification with POBA. The modification was done with $0.5 \mathrm{~mm}$ smaller diameter of conventional balloon than that of the PEB. And then, PEB angioplasty was performed with 30 seconds of inflation time as recommended. Special care was taken to avoid balloon inflation on normal segment and potential geographical miss. The procedure was completed when the result met the criteria of angiographic success (TIMI 3 flow in the main and with a diameter stenosis $<10 \%$ respectively).

All patients were treated with aspirin $100 \mathrm{mg}$ and a $300 \mathrm{mg}$ loading-dose of clopidogrel within 12 hours before procedure, respectively. Heparin was administered intravenously to maintain an activated clotting time > $250 \mathrm{sec}$ during the procedure. Administration of glycoprotein Ilb/IIla inhibitors was left to the physician's discretion. Aspirin was continued indefinitely after the procedure and clopidogrel $(75 \mathrm{mg} /$ day) was maintained to months only.

\section{IVUS analysis}

All IVUS studies were performed after intracoronary administration of $200 \mu \mathrm{g}$ of nitroglycerin using a commercial IVUS scanner (Boston Scientific Corporation, Maple Grove, MN and or Volcano Corporation, CA, USA). The IVUS catheter was advanced beyond the target ISR lesion followed by automatic transducer pull back (at $0.5 \mathrm{~mm} /$ second) to the proximal reference vessel of ISR lesion. IVUS images were recorded digitally onto a CD or DVD for offline analysis.

IVUS images were analyzed at 6 locations: Proximal and distal reference segment within $3 \mathrm{~mm}$ beyond the stent edge, proximal and distal segment of stent, the site of the narrowest neo-intimal lumen (minimal lumen area, MLA), and the site of the narrowest stent area (minimal stent area, MSA), respectively. Proximal and distal reference segments were the most normal looking cross sections within $3 \mathrm{~mm}$ beyond the stent edge, and proximal and distal segment of stent were most normal looking cross sections of proximal and distal half of stent, respectively.

Qualitative analysis was performed blind to clinical data according to the criteria of the American College of Cardiology clinical expert consensus document on IVUS. Using the planimetry software (TapeMeasure ${ }^{\circledR}$, INDEC Systems, Mountain View, California), we measured external elastic membrane (EEM) CSA (cross sectional area) $\left(\mathrm{mm}^{2}\right)$, lumen CSA $\left(\mathrm{mm}^{2}\right)$ and stent area (SA, $\left.\mathrm{mm}^{2}\right)$. Plaque burden, defined as plaque and media (P\&M) CSA, was calculated as EEM CSA minus lumen CSA [EEM CSA- lumen CSA, $\left.\left(\mathrm{mm}^{2}\right)\right]$. Percent plaque burden was calculated as P\&M CSA divided by EEM CSA [(EEM CSAlumen CSA)/ EEM CSA x 100, (\%)].

Neo-intimal CSA was calculated as stent area (SA) minus neo-intimal lumen CSA [SA-neo-intimal lumen $\left.\operatorname{CSA}\left(\mathrm{mm}^{2}\right)\right]$. Percentage of neointimal hyperplasia was calculated as neo-intimal CSA divided by SA [(SAneo-intimal lumen CSA)/SA $\times 100$, (\%)]. Stent underexpansion was defined if SA was less than $5 \mathrm{~mm}^{2}$.

\section{Primary endpoints}

PEB failure was the primary outcome of this 
study, defined if any major adverse cardiovascular events (MACE) occurred during follow-up period. A composite of MACE was defined as all deaths, any myocardial infarction, symptom driven target vessel revascularization including percutaneous coronary intervention and coronary artery bypass surgery.

\section{Statistical analysis}

Continuous variables were presented as the mean \pm standard deviations and compared with student unpaired $t$ test. Categorical variables were expressed as frequencies and percentages and compared with chisquare test. $P$ values $<0.05$ were considered significant. Statistical analysis was performed with Stat-View 5.0 (SAS Institute, Cary, North Carolina).

\section{Results}

\section{Study population and baseline clinical character- istics}

Between October 2011 and October 2013 a total of
58 consecutive patients treated with PEB [diameter: 3.0 \pm 0.4 , length: $21.9 \pm 4.8 \mathrm{~mm}$ )] for ISR were included in this study. The mean age of population was $64.5 \pm 11.4$ years, and $46(79.3 \%)$ were male.

Clinical presentations at index procedure were various: 30 patients presented as stable angina, 20 patients as unstable angina and 8 patients as non-ST-segment elevation myocardial infarction (NSTEMI). There were 8 patients (13.8\%) with BMS restenosis, 26 patients (44.8\%) with first generation DES restenosis and 24 patients (41.4\%) with second generation DES restenosis, with the mean duration from previous PCI to PEB angioplasty, as 9.4 years, 3.1 years and 2.65 years, respectively.

PEB failure was developed at 8 patients (13.8\%) after $\mathrm{PCl}$, and the mean duration from $\mathrm{PCl}$ to $\mathrm{PEB}$ angioplasty was 0.81 years (range, 0.5 years to 2.1 years). The baseline clinical demographics, procedural characteristics of index procedure and PEB angioplasty, and clinical outcomes of each group are presented in Table 1.

Table 1: Distribution of baseline clinical characteristics, procedural characteristics of index procedure and paclitaxel-eluting balloon (PEB) angioplasty, and clinical outcomes according to the presence or absence of events after PEB angioplasty $(n=58)$.

\begin{tabular}{|c|c|c|c|}
\hline \multirow{2}{*}{ Variables } & Non-PEB failure $(n=50)$ & PEB failure $(n=8)$ & \multirow{2}{*}{$P$-value } \\
\hline & \multicolumn{2}{|l|}{ mean \pm SD or $n(\%)$} & \\
\hline Sex, male & $40(80)$ & $6(75)$ & 0.62 \\
\hline Age, years & $64.7 \pm 12.1$ & $63.5 \pm 7.2$ & 0.10 \\
\hline \multicolumn{4}{|l|}{ Cardiovascular risk factors } \\
\hline Diabetes mellitus & $18(36)$ & $4(50)$ & 0.74 \\
\hline Hypertension & $30(60)$ & $8(100)$ & 0.16 \\
\hline Dyslipidemia & $42(84)$ & $8(100)$ & 0.48 \\
\hline Prior stroke & $0(0.0)$ & $0(0.0)$ & - \\
\hline Chronic kidney disease & $2(4.0)$ & $0(0.0)$ & 0.86 \\
\hline Current smoker & $4(8.0)$ & $0(0.0)$ & 0.24 \\
\hline \multicolumn{4}{|l|}{ Index procedural variables } \\
\hline Clinical presentation & & & 0.24 \\
\hline Stable angina & $28(56)$ & $2(25)$ & \\
\hline Unstable angina & $14(28)$ & $6(75)$ & \\
\hline NSTEMI & $8(16)$ & $0(0.0)$ & \\
\hline STEMI & $0(0.0)$ & $0(0.0)$ & \\
\hline Stent type & & & 0.68 \\
\hline Bare metal stent & $8(16)$ & $0(0.0)$ & \\
\hline $1^{\text {st }}$ generation DES & $20(40)$ & $4(50)$ & \\
\hline $2^{\text {nd }}$ generation DES & $22(44)$ & $4(50)$ & \\
\hline Stent diameter, $\mathrm{mm}$ & $3.1 \pm 0.3$ & $3.3 \pm 0.3$ & 0.87 \\
\hline Stent length, $\mathrm{mm}$ & $24.5 \pm 5.5$ & $20.0 \pm 3.5$ & 0.38 \\
\hline \multicolumn{4}{|c|}{ Procedural variables of PEB angioplasty } \\
\hline Diameter, mm & $3.0 \pm 0.4$ & $3.1 \pm 0.3$ & 0.53 \\
\hline Length, $\mathrm{mm}$ & $22.0 \pm 5.0$ & $21.5 \pm 3.0$ & 0.43 \\
\hline Time to TLR/TVR, years & 0.74 & 1 & 0.06 \\
\hline Clinical outcomes & & & $<0.001$ \\
\hline All-cause mortality & $0(0.0)$ & $1(12.5)$ & \\
\hline Myocardial infarction & $0(0.0)$ & $1(12.5)$ & \\
\hline TVR & $0(0.0)$ & $6(75.0)$ & \\
\hline
\end{tabular}

${ }^{*} \chi^{2}$-test and student $t$ test for no event group vs. event group; $p$-value was calculated by $\chi^{2}$ - test for continuous variables and student $t$ test for categorical variables ( $p$-value < 0.05). DES: Drug-eluting stents, ISR: In-stent restenosis, MI: Myocardial infarction, NSTEMI: Non-ST elevation myocardial infarction, PCl: Percutaneous coronary intervention, PEB: Paclitaxel-eluted balloon, STEMI: ST elevation myocardial infarction. TLR: Target lesion revascularization, TVR: Target vessel revascularization. 


\section{Comparison of baseline characteristics, procedural characteristics}

There were no differences in the sex $(40$ (80\%) vs. $6(75 \%) ; p=0.62)$, age $(64.7 \pm 12.1$ vs. $63.5 \pm 7.2 ; p=$ $0.10)$, cardiovascular risk factors; diabetes $(p=0.74)$, hypertension $(p=0.16)$, dyslipidemia $(p=0.48)$, prior angina or myocardial infarction $(p=0.24)$ and chronic kidney disease $(p=0.86)$ between PEB failure and nonfailure group. Variables on index procedures were similar between two groups, including clinical presentation $(p=$ $0.24)$, index stent type $(p=0.68)$, diameter $(p=0.87)$ and length $(p=0.43)$. Parameters on PEB procedure did not show statistical differences on diameter $(3.0 \pm 0.4$ vs. $3.1 \pm 0.3 ; p=0.53)$, length ( $22.0 \pm 5.0$ vs. $21.5 \pm 3.0 ; p$ $=0.43$ ) and duration from index $\mathrm{PCl}$ to $\mathrm{PEB}$ angioplasty (0.74 vs. 1.0 , years; $p=0.06$ ) (Table 1 ).

\section{IVUS findings}

Grayscale IVUS quantitative measurements at the proximal reference segment, proximal segment of stent, distal segment of stent and distal reference segment were assessed consecutively in order; EEM CSA of each region was $13.9,13.6,12.4$ and $11.8 \mathrm{~mm}^{2}$, respectively, lumen CSA assessed in each region were 6.31, 5.12, 5.34 and $6.0 \mathrm{~mm}^{2}$, respectively. EEM CSA and lumen CSA of each region were not statistically different between two groups. Pre-procedural IVUS findings of each groups are shown in Table 2.

In lesions with stent; proximal segment of stent, minimum lumen site, minimum stent site and distal segment of stent, neointimal CSA was 1.41, 3.74, 3.05 and $1.14 \mathrm{~mm}^{2}$, respectively. Minimum lumen site and minimum stent site had much more plaque burden

Table 2: Intravascular Ultrasound (IVUS) findings according to the presence or absence of events after PEB angioplasty ( $n=58)$.

\begin{tabular}{|c|c|c|c|}
\hline \multirow{2}{*}{ Variables } & non-PEB failure $(n=50)$ & PEB failure $(n=8)$ & \multirow{2}{*}{$P$-value } \\
\hline & \multicolumn{2}{|l|}{ mean \pm SD or $n(\%)$} & \\
\hline \multicolumn{4}{|l|}{ Proximal reference segment } \\
\hline EEM CSA, mm² & $13.8 \pm 4.10$ & $14.4 \pm 3.40$ & 0.58 \\
\hline Lumen CSA, mm² & $6.3 \pm 1.9$ & $6.4 \pm 1.3$ & 0.21 \\
\hline P\&M CSA, mm² & $7.5 \pm 3.1$ & $8.1 \pm 3.2$ & 0.97 \\
\hline Plaque burden, $\%$ & $53.2 \pm 9.80$ & $55.0 \pm 9.30$ & 0.67 \\
\hline \multicolumn{4}{|l|}{ Proximal segment of stent } \\
\hline EEM CSA, mm² & $13.3 \pm 4.30$ & $15.4 \pm 3.40$ & 0.56 \\
\hline Stent area, $\mathrm{mm}^{2}$ & $6.4 \pm 2.1$ & $8.6 \pm 1.9$ & 0.65 \\
\hline Lumen CSA, mm² & $5.0 \pm 1.6$ & $5.9 \pm 2.4$ & 0.40 \\
\hline Neointimal CSA, mm² & $1.2 \pm 1.6$ & $2.7 \pm 1.9$ & 0.66 \\
\hline \multicolumn{4}{|l|}{ Minimum lumen site } \\
\hline EEM CSA, mm² & $13.1 \pm 4.40$ & $12.9 \pm 4.70$ & 0.60 \\
\hline Stent area, $\mathrm{mm}^{2}$ & $5.9 \pm 2.6$ & $7.0 \pm 0.6$ & 0.11 \\
\hline Lumen CSA, mm² & $2.2 \pm 0.6$ & $2.4 \pm 0.8$ & 0.18 \\
\hline Neointimal CSA, mm² & $3.6 \pm 2.3$ & $4.6 \pm 1.2$ & 0.30 \\
\hline Neointimal hyperplasia, \% & $55.7 \pm 19.4$ & $65.4 \pm 14.5$ & 0.48 \\
\hline \multicolumn{4}{|l|}{ Minimum stent site } \\
\hline EEM CSA, mm² & $13.2 \pm 3.90$ & $14.3 \pm 4.20$ & 0.91 \\
\hline Stent area, $\mathrm{mm}^{2}$ & $5.5 \pm 2.7$ & $6.7 \pm 0.3$ & 0.06 \\
\hline Lumen CSA, mm² & $2.5 \pm 0.8$ & $2.8 \pm 0.9$ & 0.52 \\
\hline Neointimal CSA, mm² & $2.9 \pm 0.8$ & $4.0 \pm 0.7$ & 0.05 \\
\hline Neointimal hyperplasia, \% & $46.0 \pm 24.9$ & $59.4 \pm 12.0$ & 0.04 \\
\hline Stent under-expansion & $24(48)$ & $0(0)$ & 0.02 \\
\hline \multicolumn{4}{|l|}{ Distal segment of stent } \\
\hline EEM CSA, mm² & $12.3 \pm 4.40$ & $12.9 \pm 3.90$ & 0.93 \\
\hline Stent area, $\mathrm{mm}^{2}$ & $6.4 \pm 2.4$ & $7.0 \pm 1.2$ & 0.30 \\
\hline Lumen CSA, mm² & $5.2 \pm 1.9$ & $6.2 \pm 1.3$ & 0.55 \\
\hline Neointimal CSA, mm² & $1.2 \pm 1.6$ & $0.8 \pm 0.4$ & 0.31 \\
\hline \multicolumn{4}{|l|}{ Distal reference segment } \\
\hline EEM CSA, mm² & $11.8 \pm 5.4$ & $11.7 \pm 5.4$ & 0.75 \\
\hline Lumen CSA, mm² & $6.0 \pm 2.2$ & $6.0 \pm 1.0$ & 0.14 \\
\hline $\mathrm{P} \& \mathrm{M}$ CSA, $\mathrm{mm}^{2}$ & $5.8 \pm 3.5$ & $5.6 \pm 4.4$ & 0.30 \\
\hline Plaque burden, $\%$ & $47.8 \pm 8.30$ & $41.7 \pm 18.9$ & 0.05 \\
\hline Lesion length & $12.8 \pm 7.10$ & $19.0 \pm 3.20$ & 0.34 \\
\hline
\end{tabular}

${ }^{*} \chi^{2}$-test for no event group vs. event group; $p$-value was calculated by $\chi^{2}$ - test for continuous (p-value $\left.<0.05\right)$. CSA: Crosssectional area, EEM: External elastic membrane, $\mathrm{P} \& \mathrm{M}$ : Plaque and media. 
then stent edges. Neointimal plaque burden (presented as neointimal CSA in Table 2) of minimum lumen site and minimum stent site were greater in PEB failure group than in non-PEB failure group, though it failed to demonstrate statistically significant difference.

In PEB group, neointimal hyperplasia was greater than in non-PEB group, especially that of minimum stent site showed statistical difference (59.4 \pm 12.0 vs.
$46.0 \pm 24.9 \%, p=0.04$ ) , though that of minimum lumen site failed to show statistical difference $(p=0.48)$.

Meanwhile, stent under-expansion was more common in non-PEB failure group than in PEB failure group (24 (48\%) vs. $0(0 \%) ; p=0.02)$.

Figure 1 shows a representative case of ISR lesion with stent under-expansion with modest plaque burden
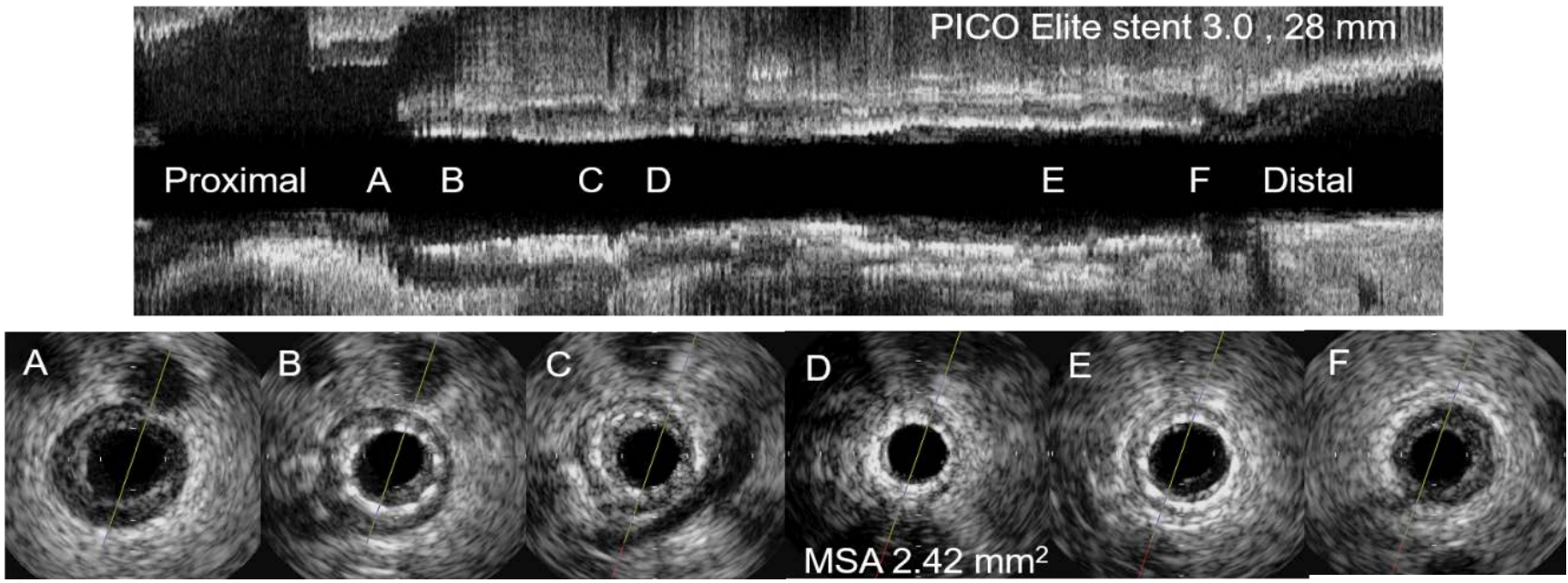

Figure 1: IVUS findings of ISR lesion with stent under-expansion.

$B, C, D)$ Pre-PCI IVUS for ISR lesion showed stent under expansion with modest plaque burden; This lesion was treated with Paclitaxel-eluting balloons $(3.0 \times 26 \mathrm{~mm})$ angioplasty, the patient was uneventful during 1.9 years of follow-up period. ISR: In-stent restenosis; PCI: Percutaneous coronary intervention; IVUS: Intravascular ultrasound; MSA: Minimal stent area.
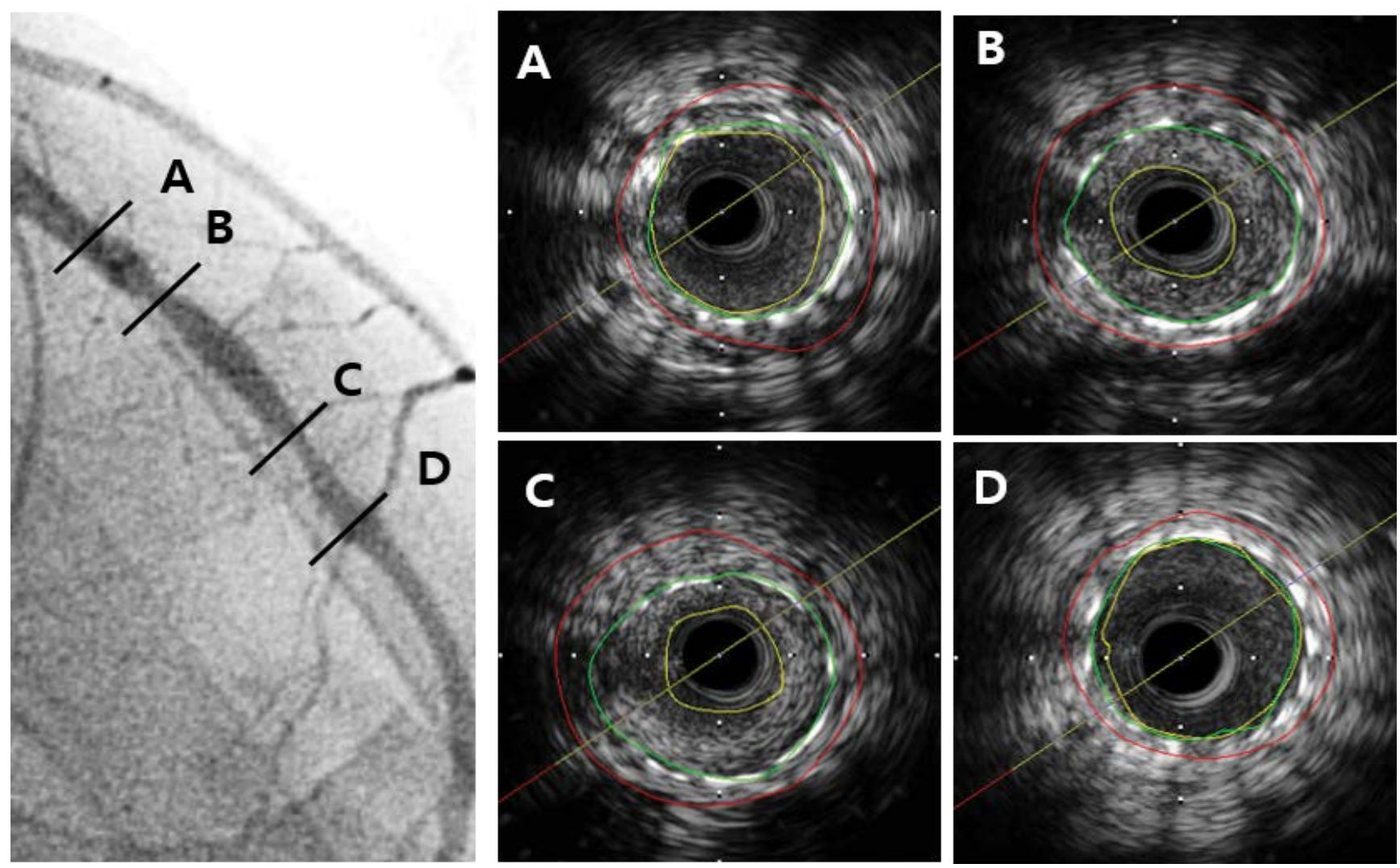

Figure 2: Representative case of ISR lesion of LAD with normally expanded stent and large plaque burden.

$\mathrm{B}, \mathrm{C})$ Pre-PCI IVUS for ISR lesion showed typical normal expansion and large plaque burden; This lesion was treated with Paclitaxel-eluting balloons $(3.0 \times 26 \mathrm{~mm})$ angioplasty, he died of sudden death 1.12 years after PEB. ISR: In-stent restenosis; LAD: Left anterior descending artery; PCI: Percutaneous coronary intervention; IVUS: Intravascular ultrasound. 
which was successfully treated with PEB. Representative cases of PEB failure with normal expansion of stent and large plaque are presented in Figure 2 and Figure 3.

\section{Discussion}

The present study investigated the IVUS characteristics predicting PEB failure in the treatment of ISR. In this study, burden of neointimal hyperplasia was higher and under-expansion of index stent was not observed in PEB failure group. On the contrary in non-PEB failure group, neointimal hyperplasia was even modest and stent under-expansion was more commonly present. This suggests that the pathophysiological mechanisms of PEB failure for ISR lesion could be associated with prominent neo-intimal hyperplasia (and its evolution) on normally extended stent.

The mechanisms of lumen gain obtained with drug-eluting balloons (DEB) are similar to those obtained by POBA, except properties regarding paclitaxel. In a previous IVUS study on POBA, Mehran R, et al. has reported that the mechanisms of balloon angioplasty were additional stent expansion and tissue extrusion out of the stent [13]. And in recent study by Alfonso, et al., mechanisms of lumen gain after balloon angiography were demonstrated as de- creased neointimal volume and stent expansion [14]. In the current study, stent under-expansion was detected frequently in non-PEB failure group, whereas none was detected in PEB failure group. This finding corresponded well to the findings of above studies. It is intuitive that PEB angioplasty can be successful in ISR lesions with stent under-expansion, because correction of under-expansion can be achieved easily by balloon angioplasty.

Regarding the neointimal hyperplasia, recent study has shown significant decrease in neointimal volume 6 month after PEB angioplasty for BMS ISR [15]. This effect might be caused by immediate direct mechanical effect of balloon angioplasty as mentioned above, and by the drug effect continuously after the intervention. Paclitaxel has been reported to have properties of rapid uptake and prolonged retention, and to cause apoptosis and necrosis of endothelial and smooth muscle cell [16]. In order that, drug delivery to the smooth muscle cell is important to achieve proper pharmacological effect on smooth muscle and to avoid endothelial toxicity.

On the contrary, recent studies have shown delayed neointimal formation after PEB therapy in DES ISR lesions $[7,17]$. This finding is similar to previous studies

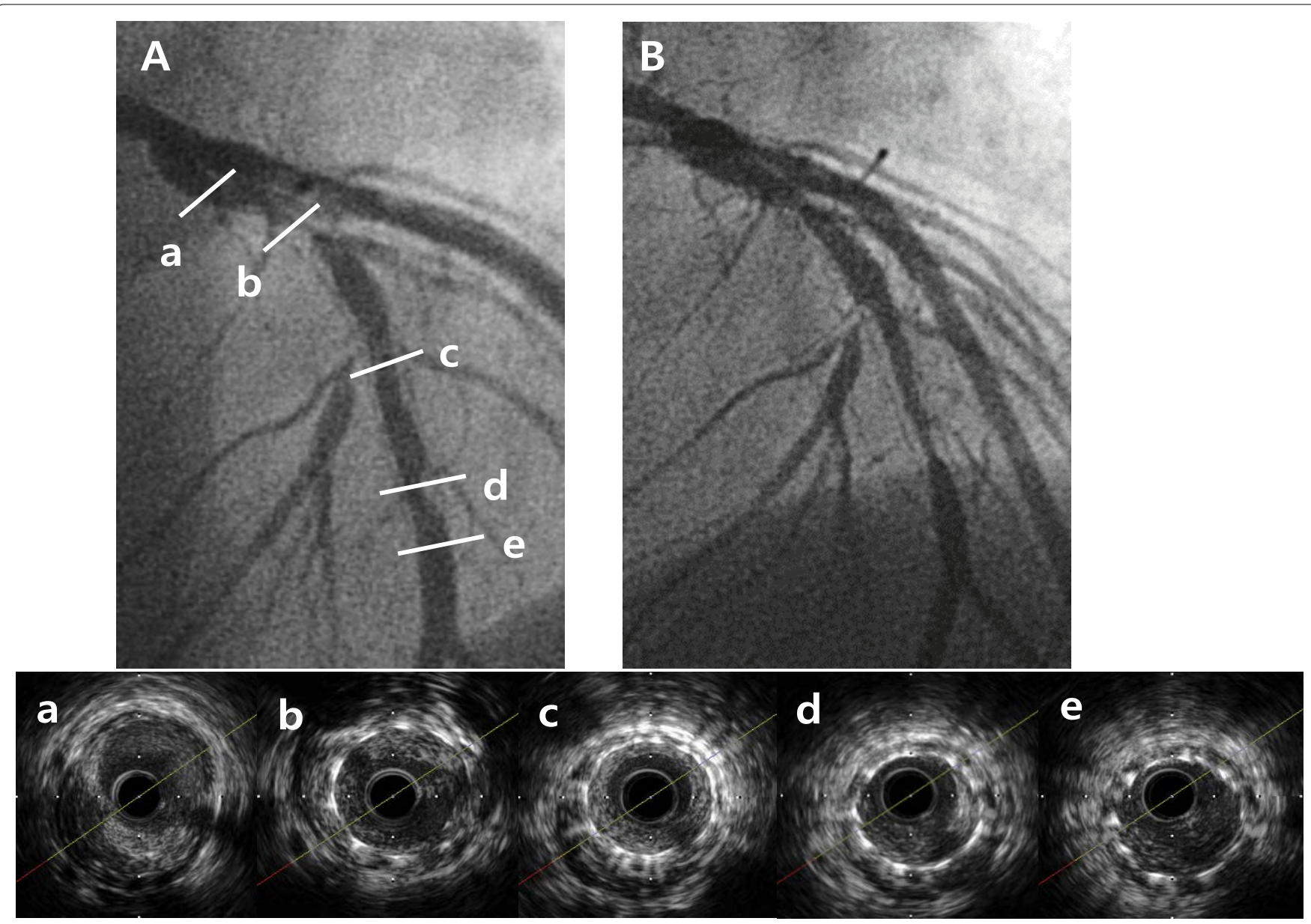

Figure 3: Representative case of ISR lesion with normally expanded stent and large plaque burden. A) Pre-PCI CAG revealed diffuse ISR of left anterior descending artery; b,c,d). Pre-PCI IVUS for ISR lesion showed typical normal expansion and large plaque burden; This lesion was treated with PEB $(3.0 \times 20 \mathrm{~mm})$ angioplasty Significant restenosis was noticed at PEB site on follow up CAG (B) 0.9 year after PEB due to ischemic chest pain. Target lesion revascularization with coronary artery bypass surgery was performed. ISR: In-stent restenosis; PCI: Percutaneous coronary intervention; CAG: Coronary angiography; IVUS: Intravascular ultrasound; PEB: Paclitaxel-eluting balloon. 
on DES, in which delayed neointimal proliferation had been reported, including experimental animal studies [18-20]. One of the possible explanations of this phenomenon is persistent drug effect-related inflammatory response caused by the drug/polymer combination $[18,21]$. In the current study, significant pre-procedural neointimal hyperplasia was demonstrated in PEB-failure group. Taking into account that the ISR of DES comprised a large proportion of the study population, this could be interpreted as that, delayed neointimal formation followed by DES has been progressed after PEB.

Future studies are warranted to investigate another $\mathrm{PCl}$ modality that might substitute PEB in the treatment of ISR lesions with prominent neointimal hyperplasia on normally extended stent.

\section{Study limitations}

In our study, post-procedural IVUS and follow-up IVUS were not performed to all patients. Changes of luminal, stent, neointimal CSA among pre-intervention, post-intervention and follow-up would provide information on nature of neointimal hyperplasia after PEB angioplasty.

Additionally in this study, only quantitative analysis on grayscale IVUS of neointimal atherosclerosis was performed. Plaque composition analysis on neointimal atherosclerosis was not performed. Recently published optical coherence tomography study on DES ISR, ISR lesions with homogeneous neointima were associated with greater subsequent regrowth of neointima compared to lesions with non-homogeneous neointima [17]. Similar approach on IVUS findings of neointimal atherosclerosis would benefit to predict clinical outcome after PEB angioplasty.

Lastly, relatively small number of patients had clinical event in current study. The low number of events resulted in limited study power to find statistically significant clinical correlates of PEB failure was difficult. Therefore it requires a multicenter trial to produce more robust results.

\section{Conclusions}

This report demonstrated the IVUS characteristics predicting PEB failure in the treatment of ISR. PEB angioplasty can be successful in ISR lesions with stent underexpansion, and can be unsuccessful in ISR lesions with significant neointimal hyperplasia on normally extended stent. Another $\mathrm{PCl}$ modality is required that might benefit in the treatment of ISR lesions with prominent neointimal hyperplasia on normally extended stent.

\section{Acknowledgement}

None.

\section{Funding Sources}

This study was supported by a grant from the Korean Society of Interventional Cardiology [\# 2010-2013].

\section{References}

1. Bonaventura K, Leber AW, Sohns C, Roser M, Boldt LH, et al. (2012) Cost-effectiveness of paclitaxel-coated balloon angioplasty and paclitaxel-eluting stent implantation for treatment of coronary in-stent restenosis in patients with stable coronary artery disease. Clin Res Cardiol 101: 573584.

2. Adriaenssens T, Dens J, Ughi G, Bennett J, Dubois C, et al. (2014) Optical coherence tomography study of healing characteristics of paclitaxel-eluting balloons vs. everolimuseluting stents for in-stent restenosis: The SEDUCE (Safety and Efficacy of a Drug elUting balloon in Coronary artery rEstenosis) randomised clinical trial. Eurolntervention 10: 439-448.

3. Chin K (2011) In-stent restenosis: The gold standard has changed. Eurolntervention 7: 43-46.

4. Alfonso F, Pérez-Vizcayno MJ, Cárdenas A, García Del Blanco B, Seidelberger B, et al. (2014) A randomized comparison of drug-eluting balloon versus everolimuseluting stent in patients with bare-metal stent-in-stent restenosis: The RIBS V Clinical Trial (Restenosis Intrastent of Bare Metal Stents: paclitaxel-eluting balloon vs. everolimus-eluting stent). J Am Coll Cardiol 63: 1378-1386.

5. Unverdorben $M$, Vallbracht $C$, Cremers $B$, Heuer $H$, Hengstenberg C, et al. (2015) Paclitaxel-coated balloon catheter versus paclitaxel-coated stent for the treatment of coronary in-stent restenosis: The three-year results of the PEPCAD II ISR study. Euro Intervention 11: 926-934.

6. Scheller B, Clever YP, Kelsch B, Hehrlein C, Bocksch W, et al. (2012) Long-term follow-up after treatment of coronary in-stent restenosis with a paclitaxel-coated balloon catheter. JACC Cardiovasc Interv 5: 323-330.

7. Rittger H, Brachmann J, Sinha AM, Waliszewski M, Ohlow $M$, et al. (2012) A randomized, multicenter, single-blinded trial comparing paclitaxel-coated balloon angioplasty with plain balloon angioplasty in drug-eluting stent restenosis: The PEPCAD-DES study. J Am Coll Cardiol 59: 1377-1382.

8. Dangas GD, Claessen BE, Caixeta A, Sanidas EA, Mintz GS, et al. (2010) In-stent restenosis in the drug-eluting stent era. J Am Coll Cardiol 56: 1897-1907.

9. Basavarajaiah S, Latib A, Shannon J, Naganuma T, Sticchi A, Bertoldi L, et al. (2014) Drug-eluting balloon in the treatment of in-stent restenosis and diffuse coronary artery disease: real-world experience from our registry. J Interv Cardiol 27: 348-355.

10. Almalla M, Schröder J, Pross V, Marx N, Hoffmann R (2014) Paclitaxel-eluting balloon versus everolimus-eluting stent for treatment of drug-eluting stent restenosis. Catheter Cardiovasc Interv 83: 881-887.

11. Basavarajaiah S, Latib A, Hasegawa T, Rezq A, Takagi K, et al. (2013) Assessment of efficacy and safety of combining "paclitaxel" eluting balloon and "limus" eluting stent in the same lesion. J Interv Cardiol 26: 259-263.

12. Mintz GS, Popma JJ, Hong MK, Pichard AD, Kent KM, et al. (1996) Intravascular ultrasound to discern device-specific effects and mechanisms of restenosis. Am J Cardiol 78: 18-22.

13. Mehran R, Mintz GS, Popma JJ, Pichard AD, Satler LF, et al. (1996) Mechanisms and results of balloon angioplasty for the treatment of in-stent restenosis. Am J Cardiol 78: 618-622.

14. Alfonso F, Sandoval J, Perez-Vizcayno MJ, Cárdenas A, 
Gonzalo N, et al. (2015) Mechanisms of balloon angioplasty and repeat stenting in patients with drug-eluting in-stent restenosis. Int J Cardiol 178: 213-220.

15. Agostoni P, Belkacemi A, Voskuil M, Nathoe HM, Doevendans PA, et al. (2013) Serial morphological and functional assessment of drug-eluting balloon for in-stent restenotic lesions: Mechanisms of action evaluated with angiography, optical coherence tomography, and fractional flow reserve. JACC Cardiovasc Interv 6: 569-576.

16. Sheehy A, Hsu S, Bouchard A, Lema P, Savard C, et al. (2012) Comparative vascular responses three months after paclitaxel and everolimus-eluting stent implantation in streptozotocin-induced diabetic porcine coronary arteries. Cardiovasc Diabetol 11: 75.

17. Lee SY, Hong MK, Shin DH, Kim JS, Kim BK, et al. (2014) Mechanisms of postintervention and nine-month luminal enlargement after treatment of drug-eluting in-stent restenosis with a drug-eluting balloon. Am J Cardiol 113: 1468-1473.

18. Wilson GJ, Polovick JE, Huibregtse BA, Poff BC (2007) Overlapping paclitaxel-eluting stents: Long-term effects in a porcine coronary artery model. Cardiovasc Res 76: 361-372.

19. Carter AJ, Aggarwal M, Kopia GA, Tio F, Tsao PS, et al. (2004) Long-term effects of polymer-based, slow-release, sirolimus-eluting stents in a porcine coronary model. Cardiovasc Res 63: 617-624.

20. Farb A, Heller PF, Shroff S, Cheng L, Kolodgie FD, et al. (2001) Pathological analysis of local delivery of paclitaxel via a polymer-coated stent. Circulation 104: 473-479.

21. Cremers B, Milewski K, Clever YP, Aboodi MS, Biedermann $M$, et al. (2012) Long-term effects on vascular healing of bare metal stents delivered via paclitaxel-coated balloons in the porcine model of restenosis. Catheter Cardiovasc Interv 80: 603-610. 